Discussion of the $\mathrm{rf}$ Parameters for RHIC

A. G. Ruggiero

May 1988 


\section{Acceleration Cycle}

This is shown in the following figure which gives the variation of the guiding dipole field $B$ versus time.

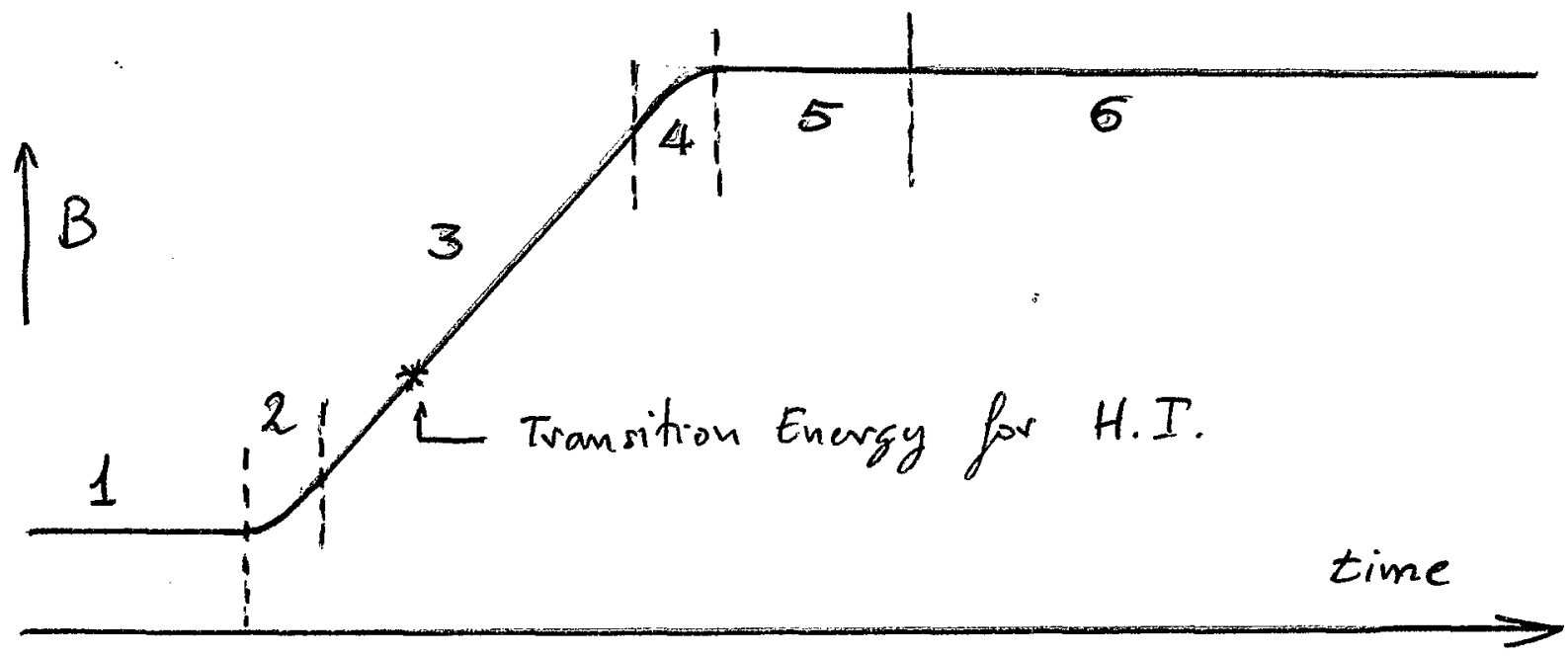

There are six well-defined time intervals.

1. Injection. The guiding field is constant. Bunches are transferred from the AGS to RHIC in a box car fashion, individually.

Only the $\mathrm{h}=342$ rf system is active, all other rf systems are "turned-off". The phase angle is $180^{\circ}$ for protons and $0^{\circ}$ for all the other heavy ions.

2. This is a transient region where $B$ and the rf parameters are varied smoothly to prepare for acceleration. We could assume a parabolic variation of $B$ over a period of $T$ seconds.

$$
B=B_{i n j}+\alpha t^{2}
$$

$0<t<T$, where $t=0$ is at beginning of this interval and $t=T$ at the end. During this period, the rf voltage and phase will change slowly in such a way that the bucket area is preserved to the value it had during injection, i.e. the previous period.

3. Next comes the period of acceleration proper where the guiding field is raised at constant rate $\dot{B}$. We shall take the acceleration 
shall see, Vsin $\phi_{S}$ is about constant. We shall also impose the condition that both $\mathrm{V}$ and $\phi_{S}$ are actually constant, except for some minor changes to accommodate for the change in velocity $\beta$ of the beam and in proximity of the transition energy crossing.

4. A second parabolic region ends the acceleration cycle. We shall assume also that it will last $T$ seconds and that the guiding field $B$ has a dependence similar to (1) except that the curvature of the B-variation is inverted. During this period the voltage and phase will have to change again slowly. We believe it is important to keep the bucket area constant during this operation at the end of which the voltage will be somewhat reduced and the phase $\phi_{\mathrm{S}}=180^{\circ}$ for all ion species, including protons.

5. During the previous intervals only the $h=342$ rf system is active, any other rf system should have been properly "turned-off". At the end of the acceleration, a period of time is spent to "rotate" or "compress" bunches in the $h=342$ rf buckets so that they can fit within narrower buckets of higher $r f$. At this point the lower rf system will be "turned-off", and it will remain "effectively off" for the remaining of the cycle, whereas the larger rf will be turned on. In this period the phase of both rf systems is $180^{\circ}$. The final voltage of the last system will be initially set to a value to provide a bucket essentially filled by the bunches.

6. Finally we have the storage mode. Only the second, larger rf system is active; the $h=342$ system will be "effectively" off for the rest of the cycle.

During the storage, the phase is $180^{\circ}$ always and the voltage can be slowly programmed, continuously increasing, to preserve the initial bunch length and to provide more bucket height to compensate for the increase of the beam momentum spread due to intrabeam scattering. 
We propose that the buckets are kept as tight as possible around the bunches throughout the acceleration cycle and the storage. In order to make the beam bunches stable against coherent instabilities, we need Landau Damping that can be provided indeed if the motion in the buckets is kept nonlinear.

\section{Capture at Injection}

Let us define the following:

Q, charge state

A, atomic mass number

$E$, total energy per atomic mass unit

$E_{O}$, rest energy per atomic mass unit

$\mathrm{S}$, longitudinal bunch area

$\gamma=\mathrm{E} / \mathrm{E}_{\mathrm{O}}=1 /{\sqrt{1-\bar{\beta}^{2}}}^{2}$

$\gamma_{\mathrm{T}}=\mathrm{E}_{\mathrm{T}} / \mathrm{E}_{\mathrm{o}}$, where

$\mathrm{E}_{\mathrm{T}}$, transition energy per atomic mass unit

$V$, peak rf voltage

$\phi_{\mathrm{S}}$, rf phase angle

$\eta=\gamma_{\mathrm{T}}^{-2}-\gamma^{-2}$

h, rf harmonic number

$R$, average radius of closed orbit

$\omega_{0}$, revolution angular frequency $=\beta c / R$

$\mathrm{Q}_{\mathrm{S}}$, number of synchrotron oscillations per period.

For a bunch area small enough compared to the bucket area B ( a factor of two would do), the following relations apply for the bunch half-eight $\Delta \mathrm{E}$ and half-length $\tau$

$$
\begin{aligned}
\Delta \mathrm{E} & =h \omega_{\mathrm{o}}\left(\frac{\mathrm{s}}{\pi} \sqrt{\frac{\mathrm{b}}{\mathrm{a}}}\right)^{1 / 2} \\
\tau & =\frac{1}{\mathrm{~h} \omega_{\mathrm{o}}}\left(\frac{\mathrm{s}}{\pi} \sqrt{\frac{\mathrm{a}}{\mathrm{b}}}\right)^{1 / 2}
\end{aligned}
$$


where

$$
\begin{aligned}
& \mathrm{a}=\eta \frac{\mathrm{h}^{2} \omega_{\mathrm{o}}^{2}}{\beta^{2} \mathrm{E}} \\
& \mathrm{b}=-\frac{\mathrm{QeV}}{2 \pi \mathrm{Ah}} \cos \phi_{\mathrm{s}}
\end{aligned}
$$

Equations (2) and (3) are valid as long as the shape of the bunches is matched to that of the rf bucket. For stability, $a$ and $b$ have the same sign, that is $\eta \cos \phi_{\mathrm{S}}<0$ always.

During transfer of the bunches from the AGS to RHIC, one requires that the shape of the buckets in the two rings are matched to each other so to provide the same dimensions $\Delta E$ and $\tau$ as given by Eqs. (2) and (3). If matching between the two rf systems does not occur, the bunches injected into RHIC will start thumbling at twice the synchrotron frequency in RHIC. This motion could cause bunch-to-bunch instabilities, create an interference with the rf loops and feedback system and eventually an increase of the bunch longitudinal area. It is expected that the tumbling motion will persist for a long time and eventually smear away in a period of time comparable to the period of the synchrotron oscillations. Thus, it is important that bunch shape is preserved during transfer from the AGS to RHIC.

Define the following quantity:

$$
x=\left[\begin{array}{cc}
-\frac{h \mathrm{~V}}{\eta \mathrm{R}^{2}} & \cos \phi_{\mathrm{S}}
\end{array}\right]^{1 / 2}
$$

After smearing, it can be easily proven that, the final bunch area $S_{\text {RHIC }}$ in RHIC is given by

$$
\mathrm{S}_{\mathrm{RHIC}}=\mathrm{S}_{\mathrm{AGS}} \times\left\{\text { the largest of } x_{\mathrm{RHIC}} / x_{\mathrm{AGS}} \text { and } x_{\mathrm{AGS}} / x_{\mathrm{RHIC}}\right\}
$$

For matching, to avoid bunch area dilution, it is simply required that the parameters of the two rings, AGS and RHIC, are adjusted so that $\chi$ has the same value for both of them. 
We shall make next few applications of these considerations. We start first with the case of transfer of proton bunches.

\subsection{Proton Bunches}

We assume the following values:

AGS : $\mathrm{R}=128.5 \mathrm{~m}$

$\mathrm{h}=12$

$\gamma_{\mathrm{T}}=8.5$

$\mathrm{V}=300 \mathrm{KV}$

$\phi_{\mathrm{S}}=180^{\circ}$

RHIC: $\mathrm{R}=610.2 \mathrm{~m}$

$\mathrm{h}=342$

$\gamma_{\mathrm{T}}=24.5$

$\phi_{\mathrm{S}}=180^{\circ}$

For a matched transfer, one requires $\mathrm{V}=12 \mathrm{kV}$ in RHIC. This value is independent of the bunch area $S$. Observe that, because several AGS pulses are required to fill RHIC over a long period of time, only stationary buckets can be provided at injection in RHIC, that is $\phi_{\mathrm{S}}=180^{\circ}$.

The only other parameter that one can change for RHIC is $h$. With all the other parameters unchanged we have

$\begin{array}{lcl}\frac{h}{171} & \frac{\mathrm{V}, \mathrm{RHIC}}{24 \mathrm{KV}} & \frac{\mathrm{B}, \mathrm{RHIC}}{6.07 \mathrm{eV} \cdot \mathrm{sec}} \\ 342 & 12 & 1.52 \\ 684 & 6 & 0.38\end{array}$

which is suggestive of the choice of lower rf during capture in RHIC, first because it would provide a voltage level comfortable with operation and second a large bucket area (B). On the other hand $Q_{S}=0.00012$ and it is independent on the choice of $h$.

Next we give the bunch dimensions $\tau$ and $\Delta \mathrm{p} / \mathrm{p}=\Delta \mathrm{E} / \beta^{2} \mathrm{E}$ versus bunch area consistent with the AGS parameters given above. 


\begin{tabular}{|c|c|c|}
\hline $\mathrm{S}$ & $\tau$ & $\Delta_{p / p}$ \\
\hline $0.3 \mathrm{eV} \cdot \mathrm{sec}$ & $6.0 \mathrm{nsec}$ & $0.54 \times 10^{-3}$ \\
\hline 0.5 & 7.7 & 0.70 \\
\hline 1.0 & 10.9 & 0.99 \\
\hline
\end{tabular}

Other parameters for the AGS are $Q_{S}=0.00050$ and a very generous bucket area $\mathrm{B}=54.6 \mathrm{eV} \cdot \mathrm{sec}$.

In order to obtain more favorable rf parameters for RHIC, we can consider changing some of the rf parameters for the AGS. For instance, we can change $\phi_{S}$ at constant $V$

\begin{tabular}{lll}
$\mathrm{Q}_{\text {SGS }}$ & $\mathrm{V}_{\text {RHIC }}$ & $\mathrm{B}_{\text {RHIC }}$ \\
\hline $180^{\circ}$ & $12.0 \mathrm{kV}$ & $1.52 \mathrm{eV} \cdot \mathrm{sec}$ \\
150 & 10.4 & 1.41 \\
120 & 6.0 & 1.07 \\
1.00 & 2.1 & 0.63
\end{tabular}

or the voltage $\mathrm{V}\left(\phi_{\mathrm{S}}=180^{\circ}\right)$

\begin{tabular}{lll}
$\mathrm{V}_{\mathrm{AGS}}$ & $\mathrm{V}_{\mathrm{RHIC}}$ & $\mathrm{B}_{\mathrm{RHIC}}$ \\
\hline $0.1 \mathrm{MV}$ & $4.0 \mathrm{kV}$ & $0.88 \mathrm{eV} \cdot \mathrm{sec}$ \\
0.3 & 12.0 & 1.52 \\
0.6 & 24.1 & 2.14 \\
1.0 & 40.1 & 2.77
\end{tabular}

Eventually one could even consider a new if in the AGS with the following results ( $\mathrm{V}=0.3 \mathrm{MV}, \phi_{\mathrm{S}}=180^{\circ}$ in the AGS)

\begin{tabular}{llcl}
$\mathrm{h}_{\mathrm{AGS}}$ & $\mathrm{V}_{\mathrm{RHIC}}$ & $\mathrm{B}_{\mathrm{AGS}}$ & $\mathrm{B}_{\mathrm{RHIC}}$ \\
\hline 12 & $12.0 \mathrm{kV}$ & $54.62 \mathrm{eV} \cdot \mathrm{sec}$ & $1.52 \mathrm{eV} \cdot \mathrm{sec}$ \\
24 & 24.1 & 19.31 & 2.14 \\
48 & 48.1 & 6.83 & 3.03
\end{tabular}


Clearly a modification of the rf system in the AGS, either by adding more voltage or by raising the frequency altogether could be quite beneficial. At the same time it seems important that bunches are not extracted from the AGS during the ramp but from stationary buckets.

Finally there is the possibility of varying the transition energy in the AGS, whereas one would prefer to leave $\gamma_{\mathrm{T}}$ unchanged in RHIC. With all the other parameters unchanged we have

\begin{tabular}{lll}
$\gamma_{\mathrm{T}^{\mathrm{AGS}}}$ & $\mathrm{V}_{\mathrm{RHIC}}$ & $\mathrm{B}_{\mathrm{RHIC}}$ \\
\hline 8.5 & $12.0 \mathrm{kV}$ & $1.52 \mathrm{eV} \cdot \mathrm{sec}$ \\
10 & 17.2 & 1.81 \\
15 & 45.0 & 2.93 \\
20 & 104 & 4.46 \\
25 & 264 & 7.10
\end{tabular}

Larger values of $\gamma_{\mathrm{T}}$ are more favorable because they would provide shorter bunches that can fit better into the narrow buckets of RHIC.

\subsection{Heavy Ion Bunches}

For all heavy-ions, injection occurs below transition energy, thus $\phi_{\mathrm{S}}=0^{\circ}$. Assuming all the other AGS and RHIC parameters unchanged except for the AGS rf voltage which is now $200 \mathrm{kV}$ we have the following, for transfer matching

\begin{tabular}{lllll} 
Ion Species & Inject. Energy & $\mathrm{Q}_{\text {sRHIC }}$ & $\mathrm{V}_{\mathrm{RHIC}}$ & $\mathrm{B}_{\mathrm{RHIC}}$ \\
\hline Deuterium & $13.6 \mathrm{GeV} / \mathrm{amu}$ & 0.00044 & $41 \mathrm{kV}$ & $0.71 \mathrm{eV} \cdot \mathrm{sec} / \mathrm{amu}$ \\
Carbon & 13.6 & 0.00043 & 40 & 0.71 \\
Sulfur & 13.6 & 0.00042 & 39 & 0.70 \\
Copper & 12.4 & 0.00058 & 56 & 0.68 \\
Iodine & 11.2 & 0.00082 & 84 & 0.65 \\
Gold & 10.7 & 0.00095 & 101 & 0.65
\end{tabular}


The values in this table do not depend on the bunch area. With the present unchanged AGS conditions, one obtains for the case of heavy ions a more favorable rf voltage value for RHIC at capture. Nevertheless observe that the rf bucket area is small and one should make sure that the bunch area is even smaller than that, for instance a factor of two.

There is quite a variation in the voltage requirement between the cases of protons and gold ions, of about a factor of ten. of course all the considerations above for the case of proton bunches apply as well to the case of heavy ions. In particular we could adjust the value of $\gamma_{T}$ in the AGS for all the ion species considered, including protons, for the same rf voltage requirement of about $100 \mathrm{kV}$ during transfer in RHIC. The results are given in the following table where the rf voltage in the AGS is $0.3 \mathrm{MV}$ for protons and 0.2 MV for all other heavy ions. All the other parameters for AGS and RHIC are unchanged.

$\begin{array}{lcccccc} & \gamma_{\mathrm{T}} & \mathrm{B} & \mathrm{Q}_{\mathrm{S}} & \mathrm{V} & \mathrm{B} & \mathrm{Q}_{\mathrm{S}} \\ \text { Proton } & 20 & 160 \mathrm{eV} \cdot \mathrm{sec} & 0.00017 & 104 \mathrm{kV} & 4.46 \mathrm{eV} \cdot \mathrm{sec} / \mathrm{amu} & 0.00035 \\ \text { Deuterium } & 11.1 & 40 & 0.00023 & 100 & 1.11 & 0.00068 \\ \text { Carbon } & 11.2 & 40 & 0.00022 & 100 & 1.12 & 0.00068 \\ \text { Sulfur } & 11.2 & 40 & 0.00023 & 99 & 1.12 & 0.00067 \\ \text { Copper } & 10 & 32 & 0.00026 & 99 & 0.89 & 0.00077 \\ \text { Iodine } & 9 & 26 & 0.00029 & 103 & 0.72 & 0.00090 \\ \text { Gold } & 8.5 & 23 & 0.00031 & 101 & 0.65 & 0.00095\end{array}$

\section{The Acceleration Period}

Let $\dot{E}$ be the acceleration rate and $\dot{B}$ the rate of change of the guiding field. We have the following two relations

$$
\text { and } \quad \begin{aligned}
\dot{\mathrm{E}} & =\frac{\mathrm{e} \mathrm{Qc}}{2 \pi \mathrm{RA}} \quad \beta \mathrm{V} \sin \phi_{\mathrm{S}}=\mathrm{E}_{\mathrm{o}} \gamma^{3} \beta \dot{\beta} \\
\dot{\mathrm{B}} \rho & =3.13\left(1+\beta^{2} \gamma^{2}\right) \gamma \dot{\beta}
\end{aligned}
$$

In the last equation $\mathrm{B} \rho$ is given in Tesla-meter. We require $\dot{B}$ constant which, as one can see, aside from a small variation with $\beta$, implies also $V$ sin $\phi_{S}$ is constant. Within an accuracy of 0.18 , assuming a total acceleration period of 
60 seconds, we require for all ion species including protons

$$
\mathrm{V} \sin \phi_{\mathrm{S}}=47.4 \mathrm{kV} / \text { turn }
$$

The bucket area is given by

$$
\mathrm{B}=\frac{16 \mathrm{R}}{h^{2} \mathrm{c}} \alpha\left(\phi_{\mathrm{S}}\right) \sqrt{\frac{\mathrm{QeVEh}}{2 \pi \mathrm{A}|\eta|}}
$$

where $\alpha\left(\phi_{\mathrm{S}}\right)$ is the Symon-Sessler rf parameter.

We require that at beginning of the acceleration ramp the bucket area is equal to the one during injection. We also assume that during the first parabolic region the beam energy changed only by little, thus

$$
\alpha^{2} \mathrm{~V}=\mathrm{V}_{\text {injection }}=100 \mathrm{kV}
$$

Equations (4) and (5) are satisfied if

$$
\frac{\alpha^{2}}{\sin \phi_{s}}=2.1
$$

that is $\phi_{S}=12^{\circ}$ and $V=230 \mathrm{kV}$. We proposed to keep $\phi_{S}$ and $V$ constant and equal to these values.

The relation between the bucket area at top energy to the injection value can be easily derived

$$
\mathrm{B}_{\text {top }}=\mathrm{B}_{\text {inj }} \sqrt{\left(\frac{\mathrm{V} \gamma}{|\eta|}\right)_{\text {top }} \cdot\left(\frac{|\eta|}{\mathrm{V} \gamma}\right)_{\text {inj }}}
$$

The results are given in the table below. In order to preserve the bucket area during the second parabolic region, in proximity of the end of acceleration, the rf phase will be reduced from $12^{\circ}$ down to $0^{\circ}$ and the rf voltage from $230 \mathrm{kV}$ down again to $100 \mathrm{kV}$. 


$\begin{array}{lll}\text { Proton } & 250.7 \mathrm{GeV} / \mathrm{amu} & 8.02 \mathrm{eV} \cdot \mathrm{sec} / \mathrm{amu} \\ \text { Deuterium } & 124.9 & 4.06 \\ \text { Carbon } & 124.9 & 4.06 \\ \text { Sulfur } & 124.9 & 4.06 \\ \text { Copper } & 114.9 & 3.75 \\ \text { Iodine } & 104.1 & 3.41 \\ \text { Gold } & 100.0 & 3.29\end{array}$

\section{Transition Energy Crossing}

With the exception of protons, all the heavy ions have to cross transition energy in RHIC. During the crossing we assume that the rf voltage $V$ and $\phi_{S}$ do not change from their assumed values for acceleration. The rms bunch length $\sigma$ and momentum spread $\delta$ are given in the following table assuming an rms bunch area of $0.05 \mathrm{eV} \cdot \mathrm{sec} / \mathrm{amu}$. The number of ions per bunch $\mathrm{N}$ are

\begin{tabular}{|c|c|c|c|c|c|}
\hline & $\begin{array}{l}\mathrm{N} \\
\times 10^{9}\end{array}$ & $\begin{array}{l}\sigma \\
\mathrm{cm}\end{array}$ & $\begin{array}{l}\delta \\
8 \%\end{array}$ & $\begin{array}{l}\mathrm{T}_{\mathrm{ad}} \\
\mathrm{msec}\end{array}$ & $\eta_{\mathrm{O}}$ \\
\hline Deuterium & 100 & 12.5 & 0.15 & 38.6 & 5.3 \\
\hline Carbon & 22 & 13.6 & 0.15 & 38.4 & 6.9 \\
\hline Sulfur & 6.4 & 13.6 & 0.16 & 38.4 & 5.4 \\
\hline Copper & 4.5 & 13.8 & 0.15 & 40.5 & 6.6 \\
\hline Iodine & 2.6 & 14.0 & 0.15 & 43.3 & 6.6 \\
\hline Gold & 1.1 & 14.1 & 0.15 & 44.5 & 4.1 \\
\hline
\end{tabular}

equal to the values given in the CDR. As a comparison, we report the bunch dimensions for $V=1.2 \mathrm{MV}$ and $\phi_{\mathrm{S}}=23^{\circ}$, they are $\sigma=3.5 \mathrm{~cm}$ and $\delta=0.12 \%$, whereas the adiabatic period is now $\mathrm{T}_{\mathrm{ad}}=25.4 \mathrm{msec}$. That is, for gold

\begin{tabular}{lccll}
$\mathrm{V}$ & $\phi_{\mathrm{S}}$ & $\sigma$ & $\delta$ & $\mathrm{T}_{\mathrm{ad}}$ \\
\hline $1.2 \mathrm{MV}$ & $2.3^{\circ}$ & $8.1 \mathrm{~cm}$ & 0.268 & $25.4 \mathrm{msec}$ \\
0.23 & 12 & 14.1 & 0.15 & 44.5 \\
0.1 & 28.6 & 19.3 & 0.11 & 60.8
\end{tabular}


There are three problems which occurs when crossing the transition energy:

(i) The effect of the beam momentum spread on the spread of instants when actually particles cross the transition energy. To reduce this effect one requires small $\delta$, thus small voltage, for a given Vsin $\phi_{S}$, and small initial bunch area. This problem disappears for $V=100 \mathrm{kV}$ (J. Wei's numerical simulations). But it is essential that the initial rms bunch area is not larger than $0.05 \mathrm{eV} \cdot \mathrm{sec} / \mathrm{amu}$.

(ii) Mismatch due to incoherent longitudinal space charge forces. This is measured by the Sorenssen parameter $\eta_{0}$ which as one can see for an rms bunch area of $0.05 \mathrm{eV} \cdot \mathrm{sec} / \mathrm{amu}$ is quite large. This effect can be reduced by either blowing up the bunch area, as it will occur anyway during the crossing, or by reducing or cancelling the space charge longitudinal impedance $(\mathrm{Z} / \mathrm{n}=i 1.4 \mathrm{ohm})$ with an inductive wall contribution of the same amount. Otherwise numerical simulations have shown an increase of the bunch area by a factor of about 3 , that is the total bunch area, for say 958 of the beam population, is now 1.0 $\mathrm{eV} \cdot \mathrm{sec} / \mathrm{amu}$ after crossing.

(iii) Microwave instabilities. The bunches are unstable on either side of the transition energy crossing, depending on the sign of the imaginary part of $\mathrm{z} / \mathrm{n}$, but the growth rates are also relatively low and do depend on the magnitude of the imaginary part of $z / n$. Large bunch area are required to start with. It could be beneficial to have control on the coupling impedance.

\section{Individual Bunch, Microwave Instability}

We shall concentrate our calculation to the case at injection and at the end of acceleration, in both cases $\phi_{S}=0^{\circ}$ or $180^{\circ}$. The results below show the limit on the coupling impedance estimated for a $95 \%$ bunch area of 0.3 $\mathrm{eV} \cdot \mathrm{sec} / \mathrm{amu}$ and for the bunch intensities as they appear in the CDR (May 1986). 


\begin{tabular}{lll} 
& \multicolumn{2}{c}{$|\mathrm{z} / \mathrm{n}|_{\text {limit }}$} \\
\cline { 2 - 3 } & Injection & Top Energy \\
Proton & $3.9 \mathrm{ohm}$ & $1.5 \mathrm{ohm}$ \\
Deuterium & 33 & 4.3 \\
Carbon & 25 & 3.2 \\
Sulfur & 32 & 4.2 \\
Copper & 35 & 3.7 \\
Iodine & 47 & 4.6 \\
Gold & 86 & 6.8
\end{tabular}

We have also assumed that the value of the transition energy in the AGS is adjusted accordingly for each ion species as described earlier to allow operation at injection at same value of rf voltage $(100 \mathrm{kV})$. Otherwise, the limit on the coupling impedance would be lower, for instance, for protons with $\gamma_{\mathrm{T}}=8.5$ and $\mathrm{V}=12 \mathrm{kV}$ at injection $|\mathrm{Z} / \mathrm{n}|=2.3 \mathrm{ohm}$.

The stability condition on the coupling impedance is the most stringent for protons, but very likely it can be met, also considering that $|z / n|_{\text {limit }}-s^{2} / \mathrm{N}$.

\section{Bunch Compression}

The following table gives the bunch half length $r$ and half the momentum spread $\Delta \mathrm{p} / \mathrm{p}$ assuming a $95 \%$ bunch area $\mathrm{S}=0.3 \mathrm{eV} \cdot \mathrm{sec} / \mathrm{amu}, \mathrm{V}=100 \mathrm{kV}$ and $\phi_{\mathrm{S}}=180^{\circ}$ at top energy.

\begin{tabular}{lcc} 
& \multicolumn{1}{c}{$\tau$} & $\Delta \mathrm{p} / \mathrm{p}$ \\
Proton & $2.60 \mathrm{nsec}$ & $0.015 \%$ \\
Deuterium & 3.65 & 0.021 \\
Carbon & 3.65 & 0.021 \\
Sulfur & 3.65 & 0.021 \\
Copper & 3.80 & 0.022 \\
Iodine & 3.91 & 0.021 \\
Gold & 4.06 & 0.023
\end{tabular}

Both $\tau$ and $\Delta \mathrm{p} / \mathrm{p}$ increase with $\sqrt{\mathrm{S}}$. Also $\Delta \mathrm{p} / \mathrm{p}$ increases, where as $\tau$ decreases, with $\mathrm{v}^{1 / 4}$. 
It is required that the rms interaction length during beam-beam collision is $20 \mathrm{~cm}$. For head-on collision this requires in turn that the rms bunch length is $28 \mathrm{~cm}$ for all ion species. To avoid a diffusion in the length of the bunch, for instance as it could be caused by intrabeam scattering, it is useful to keep the rf buckets full. It is not clear though what is the relationship between the bucket length and the rms bunch length. The wisdom of the moment is to take a factor 5 for the ratio of these two quantities. Thus the required bucket length is $140 \mathrm{~cm}$. If the associated rf is made to correspond to an harmonic number $n \times 6 \times 57$ where $n$ is an integer, than one should chose $\mathrm{n}=8$, that is $214 \mathrm{MHz}$.

If one chose a lower frequency like $n=6$, or $160 \mathrm{MHz}$, than the bucket length is $187 \mathrm{~cm}$. For a full bucket, and assuming the scaling factor of 5 between bucket length and rms bunch length, than the rms bunch length $\sigma=37.4$ cm which, for head-on collision, gives an rms value of the interaction length of $26.5 \mathrm{~cm}$, longer than needed. To compress the bunch length to an rms value of $28 \mathrm{~cm}$, the bucket is only partially filled, and in this case one would lose the advantage of the presence of the rigid walls of the rf bucket itself.

These considerations could be quite dangerous. They assume that it is possible somehow to compare a gaussian distribution with infinite long tails with the shape of a bunch within rigid walls like those of rf buckets. Trying to define an rms bunch length is therefore somewhat ambiguous and subject to a variety of interpretations which can grossly affect its values.

Two schemes have been proposed to squeeze the bunch to a length which fit the larger rf chosen for the storage mode: bunch rotation and bunch compression. We shall consider here only the second method, where the rf voltage at $h=342$ is raised slowly until the bunch is compress to the desired length.

The following table gives the voltage in MV of the $h=342$ system to obtain different total bunch (half) lengths assuming $S=0.3 \mathrm{eV} \cdot \mathrm{sec} / \mathrm{amu}$ 


\begin{tabular}{llllll}
\multicolumn{1}{c}{$\mathrm{n}$} & 4 & 5 & 6 & 7 & 8 \\
$\tau$, nsec & 4.7 & 3.7 & 3.1 & 2.7 & 2.3 \\
\hline Proton & 0.01 & 0.025 & 0.05 & 0.09 & 0.15 \\
Deuterium & 0.04 & 0.08 & 0.17 & 0.4 & 0.7 \\
Carbon & 0.035 & 0.08 & 0.18 & 0.4 & 0.7 \\
Sulfur & 0.035 & 0.08 & 0.2 & 0.4 & 0.7 \\
Copper & 0.04 & 0.1 & 0.2 & 0.4 & 0.7 \\
Iodine & 0.05 & 0.12 & 0.25 & 0.5 & 1 \\
Gold & 0.06 & 0.14 & 0.3 & 0.5 & 1
\end{tabular}

Interesting enough, in several cases, like protons, one requires lowering the rf voltage to obtain the proper bunch length. For each choice $n$, there is a range of a factor 6 between proton and gold. The case $n=8$ (214 $\mathrm{MHz}$ ), requires a large voltage. Case $\mathrm{n}=6(160 \mathrm{MHz})$ requires a total of $300 \mathrm{kV}$ to satisfy all modes of operation. We prefer this case because it provides a most favorable range of voltages from the operation point of view. Unfortunately, we suspect that it may not provide an rms bunch length as short as required.

Once the bunch has been compressed to the desired length, the $h=342$ rf system will be turned off slowly. At the same time the large rf will be turned on to a final voltage which will provide new rf buckets tightly surrounding the beam bunches. The criterion we have chosen is to provide a bucket with an area slightly larger than the bunch area $\mathrm{S}=0.3 \mathrm{eV} \cdot \mathrm{sec} / \mathrm{amu}$. The required voltages for the second rf system are given in the table below with the synchrotron tune $Q_{S}$.

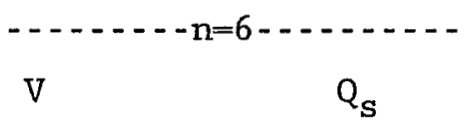

$\begin{array}{lllll}\text { Proton } & 0.035 \mathrm{MV} & 0.00027 & 0.08 \mathrm{MV} & 0.00048 \\ \text { Deuterium } & 0.12 & 0.00050 & 0.3 & 0.00091 \\ \text { Carbon } & 0.12 & 0.00050 & 0.3 & 0.00091 \\ \text { Sulfur } & 0.12 & 0.00050 & 0.3 & 0.00091 \\ \text { Copper } & 0.15 & 0.00056 & 0.35 & 0.00098 \\ \text { Iodine } & 0.2 & 0.00064 & 0.4 & 0.00105 \\ \text { Gold } & 0.2 & 0.00064 & 0.45 & 0.00111\end{array}$


Unfortunately there is still a large range of required voltage to accommodate all the modes of operation with different ion species.

\section{Storage Mode}

During storage the bunch momentum spread will grow because of intrabeam scattering, at larger rate for gold ions and at lesser rate for protons. We can estimate the growth of the rms bunch spread, but we really do not know of the relation between bucket height and rms spread for an optimum 1ifetime. As a matter of fact we do not know how to estimate the lifetime itself. We customarily take a factor of 5 between the full bucket height and rms spread. We should continue to do so, waiting for better estimates.

The momentum diffusion is a slow process and should be "measurable". The rf voltage can then be adjusted upward, continuously according to the need and the criterion chosen. Assuming that the bunch length is now fixed, only the beam momentum spread will grow and the bunch area will grow proportionally. The increase in voltage will compensate for this growth so that the bucket is always "ful1", which is the only way to preserve the bunch length.

As an example, a total voltage of 6 MV can allow a momentum spread increase for gold of a factor 4 for $n=8$, and of a factor 5 for $n=6$. 